\title{
CERTAIN CLASSES OF MEROMORPHICALLY MULTIVALENT FUNCTIONS WITH FIXED ARGUMENT OF COEFFICIENTS
}

\author{
NAK EUN CHO AND SOON YOUNG WOO
}

\begin{abstract}
In this paper, we consider some classes of meromorphically multivalent functions with fixed argument of coefficients. In those classes, we determine coefficient estimates, distortion theorems and extreme points.
\end{abstract}

\section{Introduction}

Let $\Sigma_{p}$ denote the class of functions $f$ of the form

$$
f(z)=\frac{1}{z^{p}}+\sum_{n=1}^{\infty} a_{n} z^{n} \quad(p \in N=\{1,2, \ldots\})
$$

which are analytic in $U-\{0\}$, where $U=\{z:|z|<1\}$. For analytic functions $f$ and $g$, we say that $f$ is subordinate to $g$, written $f \prec g$, if there exists a Schwarz function $w$ such that $f(z)=g(w(z))$ for $z \in U$.

Definition 1.1. Let $\Sigma_{p}^{\theta}(A, B)$ denote the class of functions $f$ of the the form (1.1) such that

$$
-z^{p+1} f^{\prime}(z) \prec p \frac{1+A z}{1+B z}
$$

and $\arg a_{n}=\theta$ for $n \in \mathbb{N}$, where $0 \leq B \leq 1$ and $-B \leq A<B(B \neq 1$ or $\cos \theta>0)$.

We note that every function $f$ belonging to the class $\Sigma_{p}^{\theta}(A, B)$ can be written as in the form

$$
f(z)=\frac{1}{z^{p}}+e^{i \theta} \sum_{n=1}^{\infty}\left|a_{n}\right| z^{n} \quad(z \in U-\{0\}) .
$$

Definition 1.2. Let $\widetilde{\Sigma}_{p}^{\theta}(A, B)$ denote the class of functions $f$ of the form (1.3) satisfying the following condition

$$
\sum_{n=1}^{\infty} n\left|a_{n}\right| \leq \delta(\theta, A, B)
$$

Received March 5, 1999.

1991 Mathematics Subject Classification. 30C45.

Key words and phrases. Mermorphically starlike of order $\alpha$, meromorphically convex of order $\alpha$, fixed argument. 
where

$\delta(\theta, A, B)=\frac{p(B-A)}{\sqrt{1-B^{2} \sin ^{2} \theta}+B \cos \theta}, \quad 0 \leq B \leq 1$ and $-B \leq A<B(B \neq 1$ or $\cos \theta>0)$.

Meromorphic univalent functions have been extensively studied by Clunie [1], Libera [2], Mogra, Reddy and Juneja [4], Pormmerenke [5] and others. In particular, the class $\Sigma_{p}^{0}(A, B)$ was studied by Mogra [3].

The object of the present paper is to obtain coefficient estimates, distortion theorems and extreme points for the classes of functions defined above.

\section{Coefficient Estimates}

Theorem 2.1. If a function $f$ of the form (1.3) belongs to the class $\Sigma_{p}^{\theta}(A, B)$, then it satisfies the condition (1.4).

Proof. Let $f \in \Sigma_{p}^{\theta}(A, B)$. By Definition (1.1), we obtain

$$
-z^{p+1} f^{\prime}(z)=p \frac{1+A w(z)}{1+B w(z)},
$$

where $w$ is an analytic function in $U$ such that $w(0)=0$ and $|w(z)|<1$ for $z \in U$. Thus we have

$$
\left|\frac{z^{p+1} f^{\prime}(z)+p}{A p+B z^{p+1} f^{\prime}(z)}\right|=|w(z)|<1 \text {. }
$$

Then we have

$$
\left|\sum_{n=1}^{\infty} n\right| a_{n}\left|z^{n+p}\right|<\left|A p+B z^{p+1} f^{\prime}(z)\right| .
$$

Putting $z=r(0<r<1)$, we obtain

$$
|w|<\left|(B-A) p-B e^{i \theta} w\right|
$$

where

$$
w=\sum_{n=1}^{\infty} n\left|a_{n}\right| r^{n+p} .
$$

Since $w$ is a real number, by (2.1) we have

$$
\left(1-B^{2}\right) w^{2}+2 p B(B-A) \cos \theta w-p^{2}(B-A)^{2}<0 .
$$

Solving this inequality with respect to $w$, we obtain

$$
\sum_{n=1}^{\infty} n\left|a_{n}\right| r^{n+p}<\delta(\theta, A, B)
$$


where $\delta(\theta, A, B)$ is defined by (1.5). Therefore, letting $r \rightarrow 1^{-}$, we have (1.4).

From Theorem 2.1, we have

Corollary 2.1. $\Sigma_{p}^{\theta}(A, B) \subset \widetilde{\Sigma}_{p}^{\infty}(A, B)$.

Corollary 2.2. If a function $f$ of the form (1.3) belongs to the class $\widetilde{\Sigma}_{p}^{\theta}(A, B)$, then

$$
\left|a_{n}\right| \leq \frac{\delta(\theta, A, B)}{n} \quad(n \in N) .
$$

The result is sharp for the extremal functions $f_{n}$ of the form

$$
f_{n}(z)=\frac{1}{z^{p}}+e^{i \theta} \frac{\delta(\theta, A, B)}{n} z^{n} \quad(n \in N) .
$$

By Corollary 2.1 and Corollary 2.2, we obtain

Corollary 2.3. If a function $f$ of the form (1.3) belongs to the class $\Sigma_{p}^{\theta}(A, B)$, then

$$
\left|a_{n}\right| \leq \frac{\delta(\theta, A, B)}{n} \quad(n \in N),
$$

where $\delta(\theta, A, B)$ is defined by (1.5). The result is sharp for $\theta=0$. The extremal functions are functions $f_{n}$ of the form

$$
f_{n}(z)=\frac{1}{z^{p}}+\frac{p(B-A)}{(1+B) n} z^{n} \quad(n \in N) .
$$

\section{Distortion Theorems and Extreme Points}

Theorem 3.1. If $f \in \Sigma_{p}^{\theta}(A, B)$, then

$$
\frac{1}{|z|^{p}}-\delta(\theta, A, B)|z| \leq|f(z)| \leq \frac{1}{|z|^{p}}+\delta(\theta, A, B)|z|
$$

and

$$
\frac{p}{|z|^{p+1}}-\delta(\theta, A, B) \leq\left|f^{\prime}(z)\right| \leq \frac{p}{|z|^{p+1}}+\delta(\theta, A, B),
$$

where $\delta(\theta, A, B)$ is defined by (1.5). The result is sharp for $\theta=0$. The extremal function is function $f_{1}$ of the form (2.3).

Proof. Let a function $f$ of the form (1.3) belong to the class $\Sigma_{p}^{\theta}(A, B)$. By Theorem 2.1 , we obtain

$$
\sum_{n=1}^{\infty}\left|a_{n}\right| \leq \sum_{n=1}^{\infty} n\left|a_{n}\right| \leq \delta(\theta, A, B)
$$


Since

$$
|f(z)|=\left|\frac{1}{z^{p}}+e^{i \theta} \sum_{n=1}^{\infty}\right| a_{n}\left|z^{n}\right| \leq \frac{1}{|z|^{p}}+\sum_{n=1}^{\infty}\left|a_{n}\right||z|^{n} \leq \frac{1}{|z|^{p}}+|z| \sum_{n=1}^{\infty}\left|a_{n}\right| \leq \frac{1}{|z|^{p}}+|z| \delta(\theta, A, B),
$$

and

$$
|f(z)|=\left|\frac{1}{z^{p}}+e^{i \theta} \sum_{n=1}^{\infty}\right| a_{n}\left|z^{n}\right| \geq \frac{1}{|z|^{p}}-\sum_{n=1}^{\infty}\left|a_{n}\right||z|^{n} \geq \frac{1}{|z|^{p}}-|z| \sum_{n=1}^{\infty}\left|a_{n}\right| \geq \frac{1}{|z|^{p}}-|z| \delta(\theta, A, B)
$$

by (3.3), we obtain (3.1). Using (3.3), we prove the estimation (3.2) analogously.

Theorem 3.2. Let $\delta(\theta, A, B)$ be defined by (1.5) and let

$$
f_{0}(z)=\frac{1}{z^{p}}
$$

and

$$
f_{n}(z)=\frac{1}{z^{p}}+e^{i \theta} \frac{\delta(\theta, A, B)}{n} z^{n} \quad(n \in N) .
$$

Then a function $f$ belongs to the class $\widetilde{\Sigma}_{p}^{\theta}(A, B)$ if and only if it is of the form

$$
f(z)=\sum_{n=0}^{\infty} \gamma_{n} f_{n}(z) \quad(z \in U-\{0\})
$$

where $\sum_{n=0}^{\infty} \gamma_{n}=1$ and $\gamma_{n} \geq 0(n \in N \cup\{0\})$.

Proof. Let a function $f$ of the form (1.3) belong to the class $\widetilde{\Sigma}_{p}^{\theta}(A, B)$. Put

$$
\gamma_{n}=\frac{n}{\delta(\theta, A, B)}\left|a_{n}\right| \quad(n \in N)
$$

and

$$
\gamma_{0}=1-\sum_{n=1}^{\infty} \gamma_{n}
$$

By the assumption and Definition 1.2, we have $\gamma_{n} \geq 0(n \in N)$ and $\gamma_{0} \geq 0$. Thus

$$
\begin{aligned}
\sum_{n=0}^{\infty} \gamma_{n} f_{n}(z) & =\gamma_{0} f_{0}(z)+\sum_{n=1}^{\infty} \gamma_{n} f_{n}(z) \\
& =\left(1-\sum_{n=1}^{\infty} \gamma_{n}\right) \frac{1}{z^{p}}+\sum_{n=1}^{\infty} \frac{n}{\delta(\theta, A, B)}\left|a_{n}\right|\left(\frac{1}{z^{p}}+e^{i \theta} \frac{\delta(\theta, A, B)}{n} z^{n}\right) \\
& =\frac{1}{z^{p}}-\sum_{n=1}^{\infty} \frac{n}{\delta(\theta, A, B)}\left|a_{n}\right| \frac{1}{z^{p}}+\sum_{n=1}^{\infty} \frac{n}{\delta(\theta, A, B)}\left|a_{n}\right| \frac{1}{z^{p}}+e^{i \theta} \sum_{n=1}^{\infty}\left|a_{n}\right| z^{n} \\
& =f(z)
\end{aligned}
$$


and the condition (3.6) follows. Conversely, let the function $f$ satisfy (3.6). Since

$$
\begin{aligned}
f(z) & =\sum_{n=0}^{\infty} \gamma_{n} f_{n}(z) \\
& =\gamma_{0} f_{0}(z)+\sum_{n=1}^{\infty} \gamma_{n} f_{n}(z) \\
& =\left(1-\sum_{n=1}^{\infty} \gamma_{n}\right) \frac{1}{z^{p}}+\sum_{n=1}^{\infty}\left(\frac{1}{z^{p}}+e^{i \theta} \frac{\delta(\theta, A, B)}{n} z^{n}\right) \gamma_{n} \\
& =\frac{1}{z^{p}}+e^{i \theta} \sum_{n=1}^{\infty} \frac{\delta(\theta, A, B)}{n} \gamma_{n} z^{n},
\end{aligned}
$$

we can write the function $f$ in the form (1.3), where

$$
\left|a_{n}\right|=\frac{\delta(\theta, A, B)}{n} \gamma_{n}
$$

Moreover,

$$
\sum_{n=1}^{\infty} n\left|a_{n}\right|=\sum_{n=1}^{\infty} \gamma_{n} \delta(\theta, A, B)=\delta(\theta, A, B)\left(1-\gamma_{0}\right) \leq \delta(\theta, A, B)
$$

Thus we have $f \in \widetilde{\Sigma}_{p}^{\theta}(A, B)$, which completes the proof of our result.

By using the same method as in the proof of Theorem 3.2, we can prove the following.

Theorem 3.3. Let $f_{0}(z)=\frac{1}{z^{p}}$ and let $f_{n}(n \in N)$ be defined by (2.3). Then a function $f$ belongs to the class $\Sigma_{p}^{0}$ if and only if it is of the form (3.6).

From Theorem 3.2 and Theorem 3.3, we obtain the following two corollaries.

Corollary 3.1. $\Sigma_{p}^{0}(A, B)=\widetilde{\Sigma}_{p}^{0}(A, B)$.

Corollary 3.2. The class $\Sigma_{p}^{0}(A, B)$ is convex.

\section{Acknowledgement}

This work was partially supported by Pukyong National University (1998) and the Korea Research Foundation (Project No.: 1998-015-D00039).

\section{References}

[1] J. Clunie, On meromorphic schlicht functions, J. London Math. Soc., 34(1959), 215-216.

[2] R. J. Libera, Meromphic close-to-convex functions, Duke Math. J., 32(1965), 121-128. 
[3] M. L. Mogra, Meromorphic multivalent functions with positive coefficients, Math. Japonica, 35(1990), 1089-1098.

[4] M. L. Mogra, T. R. Reddy and O. P. Juneja, Meromorphic univalent functions with positive coefficients, Bull. Austral. Math. Soc., 32(1985), 161-176.

[5] Ch. Pommerenke, On meromorphic starlike functions, Pacific J. Math., 13(1963), 221-235.

Department of Applied Mathemeatics, Pukyong National University, Pusan 608-737, Korea. 